\title{
EL CUESTIONARIO DE AUTOCONCEPTO FÍSICO (CAF): ANÁLISIS FACTORIAL CONFIRMATORIO Y PREDICTIVO SOBRE EL RENDIMIENTO ACADÉMICO GLOBAL Y ESPECÍFICO DEL ÁREA DE EDUCACIÓN FÍSICA
}

\section{PHYSICAL SELF CONCEPT QUESTIONNAIRE: PREDICTIVE AND CONFIRMATORY FACTOR ANALYSIS OF GLOBAL AND ESPECIFIC ACADEMIC ACHIEVMENT ON PHSYCAL EDUCATION}

\author{
Francisco Pablo Holgado Tello ${ }^{1}$, José Antonio Soriano Llorca ${ }^{2}$ \\ y LEANDRO NAVAS MARTíNEZ ${ }^{3}$ \\ 1 Facultad de Psicología. UNED. Madrid \\ 2 C.C. Ntra. Sra. de los Dolores. Benidorm (Alicante) \\ ${ }^{3}$ Facultad de Educación. Universidad de Alicante. Alicante
}

\section{Resumen}

Los objetivos de este trabajo fueron examinar la estructura factorial del CAF (Cuestionario de Autoconcepto Físico) en un contexto distinto al de su creación así como averiguar las posibles relaciones de sus dimensiones con el rendimiento académico (global y en Educación Física) y si éste se puede predecir a partir de las mismas. Los participantes fueron 574 estudiantes de ESO de la Comunidad Valenciana. Se llevaron a cabo análisis factorial confirmatorio, correlacional y de vías. Los resultados obtenidos revelan que el CAF muestra adecuados índices de fiabilidad y de discriminación, así como unos índices de ajuste aceptables, aunque, el cuestionario logra un mejor ajuste y una mayor capacidad de discriminación media si se elimina un elemento del mismo. Por otro lado, se encuentran relaciones positivas entre todas las dimensiones del cuestionario y el rendimiento en Educación Física, y negativas entre algunas de las dimensiones y el rendimiento global.

\section{Palabras Clave}

Autoconcepto Físico, Rendimiento académico, Educación Física.

\begin{abstract}
The objetives of this study were to examine the factorial structure of the CAF (Questionnaire of Physical Autoconcept) in a different context of it's creation as well as to verify the possible relations of his dimensions with the academic performance (global and in Physical Education) and if it would be possible to predict from those ones. The participants were 574 students of ESO from Valencian Community. There was carried out the confirmatory factorial, correlacional and path analysis. The results obtained reveal that the CAF shows suitable indexes of reliability and average discrimination, as well as a few acceptable indexes of adjustment, though, the questionnaire achieves a better adjustment and a bigger capacity of discrimination if an element of the same one is avoided. On the other hand, they find positive relations between all the dimensions of the questionnaire and the performance in Physical Education, and negative between some of the dimensions and the global performance.
\end{abstract}

\section{Key Words}

Physical self-concept, academic performance, Physical Education. 


\section{Introducción}

Tras la formulación del modelo multidimensional del autoconcepto (Shavelson, Hubner y Stanton, 1976), se superó la idea según la cual su estructura se reducía a un factor general en el que no se diferenciaban dimensiones (Coopersmith, 1967). Actualmente, se reconoce la existencia de varios dominios en el Autoconcepto tales como el afectivo, el académico, el social, el físico, etc., que, a su vez, se subdividen en diversas dimensiones (Atienza, Balaguer, Moreno y Fox, 2004; Byrne, 1996; Elexpuru y Villa, 1992; Esnaola, Goñi y Madariaga, 2008; Fox, 1997; Goñi y Ruíz de Azúa, 2009; Harter, 1988; Marsh, 1990; Marsh y Hattie, 1996; Núñez y GonzálezPienda, 1994).

Esta investigación se centra en el autoconcepto físico que, al igual que ocurre con otros dominios del autoconcepto, está compuesto por diferentes dimensiones. La estructura del autoconcepto físico más aceptada es la que considera cuatro dimensiones (Fox y Corbin, 1989): habilidad física, condición física, atractivo físico y fuerza (Asci, Asci y Zorba, 1999; Cox, 2009; Goñi, Palacios, Zulaika, Madariaga y Ruiz de Azúa, 2002; Goñi, Ruiz de Azúa y Rodríguez, 2006; Goñi y Ruiz de Azúa, 2009; Gutiérrez, Moreno y Sicilia, 1999), aunque hay algunas dimensiones aún por delimitar (Goñi, Ruiz de Azúa y Liberal, 2004). Por otro lado, el autoconcepto físico se ha relacionado con múltiples variables: hábitos de vida, trastornos de la alimentación, práctica deportiva, su evolución durante la vida, etc. (Dieppa, Machargo, Lújan y Guillén, 2008; Esnaola, 2008; Goñi, Ruiz de Azúa y Rodríguez, 2004; Goñi y Rodríguez, 2007; Infante y Zulaika, 2009; Moreno, Cervelló y Moreno, 2008).

De los cuestionarios y escalas que valoran las dimensiones del autoconcepto físico, merecen ser destacados el Physical Self-Perception Profile (PSPP), de Fox y Corbin (1989) y Fox (2000), y el Physical Self-Description Questionnaire (PSDQ) de Marsh, Richards, Johnson, Roche y Tremayne (1994).

El PSPP es el instrumento de mayor relevancia en los últimos años (Marsh, 1997) y consta de seis escalas: condición física, competencia deportiva, atractivo físico, fuerza, autoconcepto físico general y autoconcepto general. Sus propiedades psicométricas han sido suficientemente corroboradas en varios estudios (Asci, 2005; Gutiérrez, Moreno y Sicilia, 1999; Maïano, Ninot y Bilard, 2004; Moreno, 1997; Petrakis y Bahls, 1991) y existe una adaptación en español del PSPP, denominada Physical-Self Questionnaire (PSQ), de Moreno y Cervelló (2005), que evalúa cinco dimensiones: competencia percibida, apariencia física, condición física, fuerza y autoestima.

El PSDQ consta de once escalas: fuerza, coordinación, actividad física, grasa corporal, aptitud deportiva, apariencia física, salud, autoestima, resistencia / capacidad, autoconcepto físico global y flexibilidad. Su validez ha sido comprobada en varias investigaciones (Dunton, Jamner y Cooper, 2003; Marsh, Asci y Tomás, 2002; Tomás, 1998).

Partiendo de la versión del PSPP de Whitehead (1995), Goñi y Zulaika (2000) y Goñi et al. (2002) hacen sucesivas adaptaciones hasta obtener el Cuestionario de Autoconcepto Físico (CAF) de Goñi et al. (2006), que evalúa las siguientes dimensiones: habilidad física, condición física, fuerza física, atractivo físico, autoconcepto físico general y autoconcepto general, con adecuados índices de fiabilidad. Sin embargo, y aunque ha sido aplicado en numerosos estudios (ver Goñi, 2009), los 1344 participantes del estudio inicial (Goñi et al., 2006) eran estudiantes de ESO, Bachillerato y Universidad, con edades comprendidas entre los 11 y los 26 años y pertenecían a las provincias de Álava, Guipúzcoa, Vizcaya, Burgos y La Rioja. Por ello, cabe plantearse qué cualidades presentará el instrumento en contextos socio-culturales diferentes.

Además, como las dimensiones del autoconcepto físico, evaluadas con el CAF, se han relacionado con estilos y hábitos de vida saludable, consumo de alcohol o de tabaco, práctica deportiva, actividad física, bienestar / malestar psicológico, trastornos de la alimentación, ansiedad, etc. (Esnaola et al., 2008; Goñi et al., 2006), puede resultar de interés averiguar las posibles relaciones con el rendimiento académico y si éste se puede predecir a partir de las dimensiones del autoconcepto físico.

Así pues, dos objetivos guían este trabajo. Primero, comprobar las distintas dimensiones 
evaluadas por el CAF y sus cualidades psicométricas en un contexto diferente (el de la Comunidad Valenciana). Y, segundo, analizar las relaciones entre las distintas dimensiones del autoconcepto físico y el rendimiento académico (global y en Educación Física), aspecto que aún no se ha evaluado.

\section{Método}

\section{Participantes}

Participaron 574 estudiantes (310 chicos y 264 chicas), que cursan Educación Secundaria Obligatoria, con edades comprendidas entre los 11 y los 17 años $(M=13.07, D T=1.26)$ pertenecientes a tres centros de la Comunidad Valenciana, ubicados en la provincia de Alicante. La selección de los participantes se lleva a cabo por doble muestreo aleatorio simple (se escoge al azar una de las tres provincias de la comunidad y, de ella, al azar se seleccionan tres centros de ESO). Según Arkin y Colton (1962), el tamaño de la muestra garantiza un error muestral inferior a $\pm 5 \%$ (nivel de confianza $>95 \% ; P=.5$ ).

\section{Instrumentos}

El Cuestionario de Autoconcepto Físico (CAF) de Goñi et al. (2006), consta de 36 ítems que se agrupan en seis dimensiones o subescalas: habilidad deportiva (con un valor alfa de .84) compuesta por los ítems 1, 6, 17, 23, 28 y 33, que expresan ideas como "No tengo cualidades para los deportes» $\mathrm{O}$ «Me veo torpe en las actividades deportivas»; condición física (con un valor alfa de .88), integrada por los ítems $2,7,11,18,24$ y 29, que expresan ideas como: «Tengo mucha energía física» $\mathrm{o}$ «Puedo correr y hacer ejercicio durante mucho tiempo sin cansarme»; atractivo físico (con un valor alfa de .87), que agrupa a los ítems $8,12,19,25,30$ y 34 , con expresiones como «Me cuesta tener un buen aspecto físico» o «Siento confianza en cuanto a la imagen física que transmito»; fuerza (con un valor alfa de .83), formada por los elementos 3, 9, 13, 20, 31 y 35, e ítems como «Soy capaz de realizar actividades que exigen fuerza» o "Soy fuerte»; autoconcepto físico general (con un valor alfa de .86), que integra a las cuestiones 4, 14, 16, 21, 26 y 36, con enunciados como "Físicamente me siento peor que los demás» o "Físicamente me siento bien», y autoconcepto general (con un valor alfa de .84), agrupando a los elementos 5, 10, 15, 22, 27 y 32, que exponen ideas como «Me siento feliz» o «Desearía ser diferente». El coeficiente de fiabilidad «del cuestionario es de .93»(Goñi, 2009, p. 275).

\section{Procedimiento}

Tras requerir las autorizaciones oportunas de la dirección de los centros y de las familias de los alumnos, el cuestionario se administró en el aula habitual. Fue respondido de forma voluntaria y se instruyó a los sujetos cómo debían responder, se les solicitó la máxima sinceridad y se les garantizó la confidencialidad de los datos que se obtuvieran. Después de celebrarse las sesiones correspondientes a la primera evaluación, se recabaron las calificaciones obtenidas por los participantes a partir de las actas de evaluación, tanto en Educación Física como en el resto de las áreas.

\section{Diseño, variables y análisis de datos}

El diseño es correlacional básico ex post facto (León y Montero, 1998) ya que las variables no se manipulan intencionalmente.

Las variables a predecir fueron el rendimiento académico global, que se expresa como la nota media de todas las calificaciones obtenidas en las distintas áreas y el rendimiento académico en Educación Física, a través de la calificación en esa área. Las variables predictoras fueron las diferentes dimensiones del autoconcepto físico evaluadas con el CAF.

Los datos fueron sometidos a análisis factorial comfirmatorio, correlacionales y análisis de vías. Se han utilizado los programas estadísticos SPSS, versión 15.0, PRELIS, versión 2.30, y LISREL, versión 8.54.

\section{Resultados}

La fiabilidad del CAF medida mediante el coeficiente de consistencia interna $\alpha$ de Cronbach 
es $\alpha=.93$ y los índices de discriminación muestran que todos los elementos discriminan satisfactoriamente, de hecho, los coeficientes de discriminación se encuentran entre .668, para el ítem 11 («Estoy en buena forma física»), y .337, para el ítem 3 («Tengo más fuerza que la mayoría de la gente de mi edad»). Únicamente el ítem 32 («No tengo demasiadas cualidades como persona») obtiene un índice de discriminación por debajo de .2. En función de ello, para el resto de los análisis se ha eliminado de la escala. La discriminación media de la escala es .50.

Para llevar a cabo el análisis factorial confirmatorio con las respuestas del CAF, dado que el número de ítems es elevado, se emplea el método de mínimos cuadrados no ponderados $(U L S)$, y dado que los ítems son variables ordinales, se han usado correlaciones policóricas.

En total se obtienen 595 correlaciones (35* 34/2). Para 73 correlaciones la asunción de normalidad bivariada se rechazó a un nivel de significación del $.00008(\alpha=.05 / 630)$ que se corresponde con un valor de Chi-cuadrado de 49.9 $(g l=15)$. Por otra parte, el RMSEA fue significativamente menor que 0.1 en todos los casos, lo que justifica el uso de este tipo de correlaciones (Jöreskog, 2001).

En la tabla 1 se muestra la solución completamente estandarizada para el modelo propuesto originalmente por Goñi et al., (2006) que obtiene un valor de $\chi^{2}=2707.86(g l=545$; $p=.000)$ y los siguientes índices de ajuste: $R M S E A=.10 ; G F I=.97$ y $A G F I=.96$.

Observando los índices de modificación, encontramos algunos indicios sobre cómo mejorar el ajuste del modelo. Tras someter los índices de modificación a un análisis sustantivo se decide que el ítem 11 («Estoy en buena forma»), además de saturar en condición física, también se incluya en atractivo físico y en autoconcepto físico. El ítem 16 ( «Soy de las personas que están descontentas de cómo son físicamente») además de saturar en autoconcepto físico también lo haga habilidad física y condición física; y el ítem 27 («Estoy haciendo bien las cosas») que, originalmente saturaba en autoconcepto general, también se incluya en habilidad física y en condición física. Entendemos que la inclusión de estos nuevos parámetros está justificada teóricamente porque la formulación del ítem número 11 puede abarcar el dominio del atractivo físico y el dominio del autoconcepto físico, ya que una persona que esté en buena forma, normalmente se sentirá más atractivo físicamente y tendrá un mejor autoconcepto físico, así mismo, el ítem 16 abarca los dominios autoconcepto físico, habilidad física y condición física, pues un sujeto con una buena habilidad física y una buena condición física, percibirá un mejor autoconcepto físico. Por último, el ítem 27 puede saturar en autoconcepto general, habilidad física y condición física, ya que percibir hacer bien las cosas encaja en estas tres dimensiones.

Una vez introducidos los índices de modificación, los valores obtenidos para los distintos índices de ajuste fueron los siguientes: $\chi^{2}=$ $2439.72(g l=539 ; p=.000) ; R M S E A=.08$; $G F I=.97 ; A G F I=.97$. Lo que supone una mejora significativa en el ajuste del modelo $\left(\Delta \chi^{2}=\right.$ 268.14; $g l=6$ ). En la tabla 1 se recoge la solución completamente estandarizada del modelo resultante.

Posteriormente, se realizan análisis correlacionales (correlaciones bivariadas) con las variables latentes que resultan de los análisis factoriales confirmatorios, y de las calificaciones globales y en educación física. En la tabla 2 se muestra la matriz de coeficientes de correlación ( $r$ de Pearson). Como se puede observar en la misma, se da una compleja red de relaciones y de asociaciones entre las variables consideradas. Llama la atención que casi la mitad de los coeficientes entre las dimensiones del autoconcepto y el rendimiento académico (global y en Educación Física) no son estadísticamente significativos y los que lo son presentan valores bajos.

Para analizar con mayor profundidad las relaciones entre las distintas dimensiones del autoconcepto físico (habilidad física, condición física, atractivo físico, fuerza, autoconcepto físico general y autoconcepto general) y el rendimiento académico (global y en Educación Física), se plantea un modelo predictivo en el que las variables predictoras son las dimensiones del autoconcepto físico y el rendimiento en Educación Física y la variable criterio es el rendimiento global. El modelo se obtuvo a partir del estudio de las correlaciones entre las va- 
Tabla 1. Solución completamente estandarizada para el Cuestionario de Autoconcepto Físico.

\begin{tabular}{|c|c|c|c|c|c|c|}
\hline Items & HF & CF & $\mathbf{A F}$ & FU & AFIS & AG \\
\hline 1. Soy bueno/a en los deportes & .78 & & & & & \\
\hline 6. No tengo cualidades para los... & .70 & & & & & \\
\hline 17. Tengo más habilidad que la ... & .62 & & & & & \\
\hline 23. Soy de las personas a las que ... & .49 & & & & & \\
\hline 28. Practicando deporte soy una ... & .76 & & & & & \\
\hline 33. Me veo torpe en las ... & .61 & & & & & \\
\hline 2. Tengo mucha resistencia física & & .79 & & & & \\
\hline 7. Me quedo pronto sin aliento y ... & & .62 & & & & \\
\hline 11. Estoy en buena forma & & .48 & .74 & & -.35 & \\
\hline 18. Puedo correr y hacer .. & & .76 & & & & \\
\hline 24. En actividades como la de ... & & .65 & & & & \\
\hline 29. Tengo mucha energía física & & .81 & & & & \\
\hline 8. Me cuesta tener un buen ... & & & .55 & & & \\
\hline 12. Me siento contento/a ... & & & .82 & & & \\
\hline 19. Siento confianza en cuanto ... & & & .75 & & & \\
\hline 25 . No me gusta mi imagen... & & & .82 & & & \\
\hline 30. Soy guapo/a & & & .53 & & & \\
\hline 34. Me gusta mi cara y mi cuerpo & & & .67 & & & \\
\hline 3. Tengo más fuerza que la... & & & & .60 & & \\
\hline 9. Me cuesta levantar tanto ... & & & & .37 & & \\
\hline 13. Soy capaz de realizar ... & & & & .77 & & \\
\hline 20. Destaco en actividades en ... & & & & .83 & & \\
\hline 31. Soy fuerte & & & & .67 & & \\
\hline 35. No me veo en el grupo ... & & & & .60 & & \\
\hline 4. Físicamente me siento bien & & & & & .68 & \\
\hline 14. En lo físico me siento ... & & & & & .73 & \\
\hline 16. Soy de las personas que... & .16 & -.56 & & & .91 & \\
\hline 21. Mi cuerpo me transmite... & & & & & .68 & \\
\hline 26. No me siento a gusto ... & & & & & .69 & \\
\hline 36. Físicamente me siento ... & & & & & .74 & \\
\hline 5. Me siento a disgusto ... & & & & & & .57 \\
\hline 10. Me siento feliz & & & & & & .58 \\
\hline 15. No me gusta lo que ... & & & & & & .62 \\
\hline 22. Desearía ser diferente & & & & & & .67 \\
\hline 27. Estoy haciendo bien las cosas & .28 & .09 & & & & .25 \\
\hline
\end{tabular}

$\mathrm{HF}=$ Habilidad física; $\mathrm{CF}$ = Condición física; $\mathrm{AF}=$ Atractivo físico;

$\mathrm{FU}=$ Fuerza; AFIS = Autoconcepto físico general; AG = Autoconcepto general .

Tabla 2. Matriz de correlaciones bivariadas.

\begin{tabular}{cccccccc}
\hline & HF & CF & AF & FU & AFIS & AG & CEF \\
\hline CF & $.35^{* *}$ & & & & & & \\
AF & $.14^{* *}$ & $.37^{* *}$ & & & & & \\
FU & $.28^{* *}$ & $.47^{* *}$ & $.30^{* *}$ & & & & \\
AFIS & $.19^{* *}$ & $.23^{* *}$ & $.14^{* *}$ & $.22^{* *}$ & & & \\
AG & $.22^{* *}$ & .01 & -.00 & $.14^{* *}$ & $.33^{* *}$ & & \\
CEF & .07 & $.10^{* *}$ & $.10^{* *}$ & $.10^{*}$ & .01 & -.00 & \\
CG & -.04 & $-.10^{*}$ & .02 & $-.14^{* *}$ & -.08 & $-.10^{* *}$ & $.54^{* *}$ \\
\hline
\end{tabular}

$\mathrm{HF}=$ Habilidad física; $\mathrm{CF}=$ Condición física; $\mathrm{AF}=$ Atractivo físico;

FU = Fuerza; AFIS = Autoconcepto físico general; AG = Autoconcepto general;

$\mathrm{CEF}=$ Calificación en Educación Física; $\mathrm{CG}$ = Calificación global . 
Figura 1. Modelo correspondiente a la solución completamente estandarizada.

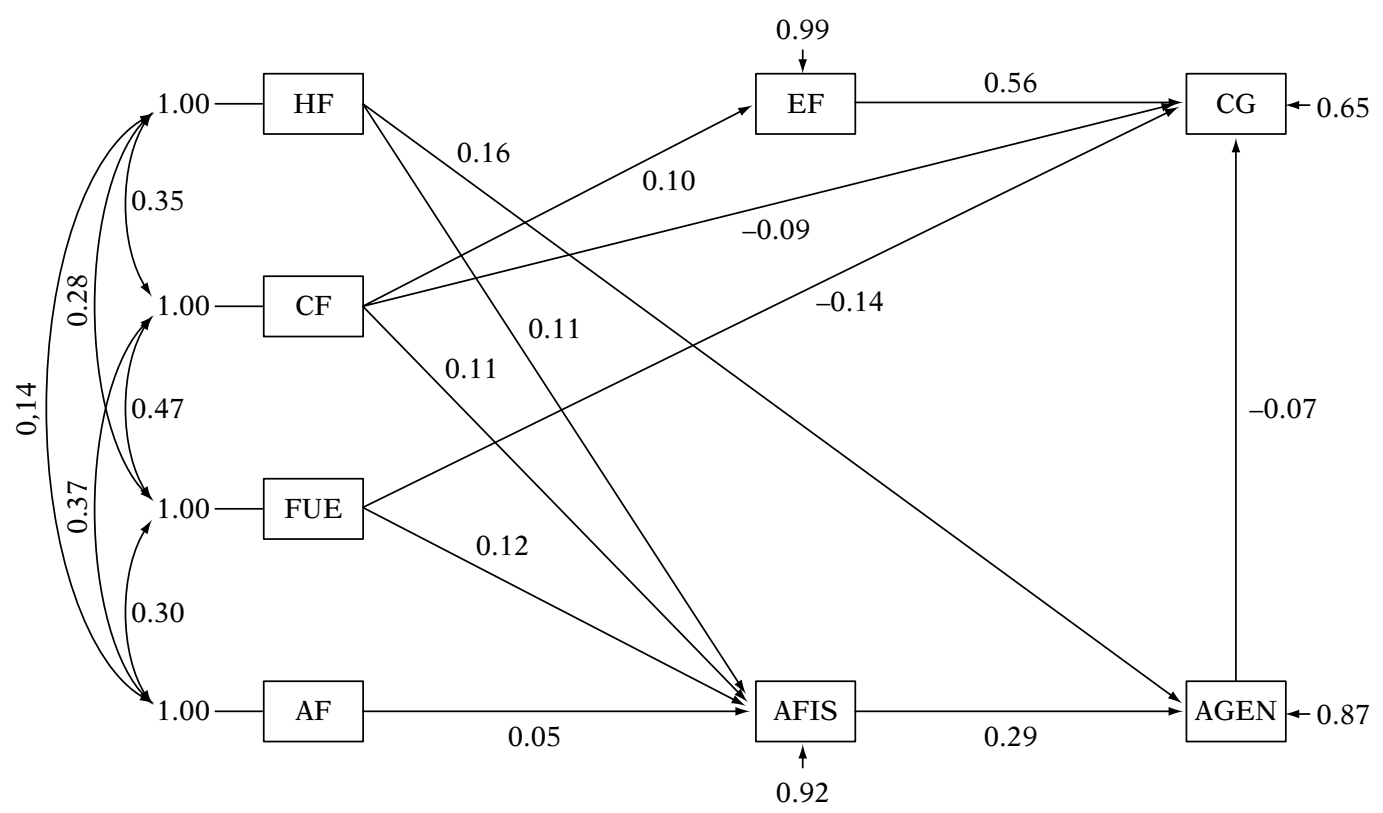

( $\mathrm{HF}$ = Habilidad física; $\mathrm{CF}$ = Condición física; $\mathrm{AF}$ = Atractivo físico; FU = Fuerza; AFIS = Autoconcepto físico; AGEN = Autoconcepto general; EF = Calificación en Educación Física; $C G$ = Calificación global).

riables y de criterios teóricos derivados de estudios previos, que relacionan autoconcepto y rendimiento académico (García, González-Pienda, Núñez, González-Pumariega, Álvarez, Roces, González y Valle, 1998; Skaalvik y Hagtvet, 1990). Los índices de ajuste correspondientes a la solución completamente estandarizada del modelo correspondiente a la figura 1 son los siguientes: $\chi^{2}=21.16 ; g l=11 ; p=.032 ; R M S E A=$ $.040 ; G F I=.99 ; C F I=.99 ; A G F I=.97$. En la tabla 3 se muestran los coeficientes Beta y Gamma correspondientes a la solución.
Los coeficientes Beta que resultan estadísticamente significativos son los correspondientes a las relaciones entre la calificación en Educación Física y la calificación global, entre el autoconcepto físico y la calificación general, y entre el autoconcepto general y la calificación global (en sentido negativo). Por su lado, los coeficientes Gamma que aparecen como significativos describen las relaciones entre la habilidad física con la calificación global y con el autoconcepto físico, la condición física con la calificación en Educación Física, con el auto-

Tabla 3. Coeficientes correspondientes a la solución completamente estandarizada.

\begin{tabular}{cccccccc}
\hline Variables & EF & AGEN & AFIS & HF & CF & FUE \\
$(\gamma)$ & $(\beta)$ & $(\beta)$ & - & $\begin{array}{c}\text { AF } \\
(\gamma)\end{array}$ \\
\hline CG & $.56^{*}$ & $-.07^{*}$ & - & - & $-.09^{*}$ & $-.14^{*}$ & - \\
EF & - & - & - & - & $.10^{*}$ & - & - \\
AGEN & - & - & $.29^{*}$ & $.16^{*}$ & - & - & - \\
AFIS & - & - & - & $.11^{*}$ & $.11^{*}$ & $.12^{*}$ & .05 \\
\hline
\end{tabular}

$\mathrm{HF}$ = Habilidad física; $\mathrm{CF}$ = Condición física; $\mathrm{AF}$ = Atractivo físico;

$\mathrm{FU}=$ Fuerza; AFIS = Autoconcepto físico; AGEN = Autoconcepto general;

$\mathrm{EF}=$ Calificación en Educación Física; $\mathrm{CG}$ = Calificación global .

*Indica los coeficientes significativamente distintos de cero. 
concepto físico y con la calificación global (en sentido negativo), y la fuerza que se relaciona en sentido negativo con la calificación global y, en sentido positivo, con el autoconcepto físico.

\section{Discusión}

En general, este artículo ha pretendido analizar las cualidades psicométricas del CAF en una muestra de estudiantes de ESO de la Comunidad Valenciana. Se analizó su fiabilidad, su capacidad de discriminación y la capacidad predictiva de las dimensiones del autoconcepto físico sobre los dominios de autoconcepto físico y autoconcepto general y, a su vez, de éstos sobre el rendimiento académico en Educación Física y sobre el rendimiento global. Todo ello puede ser de interés porque hay pocos estudios que analicen las relaciones entre las dimensiones y dominios del Autoconcepto Físico y el rendimiento académico (Marsh, Chanal y Sarrazin, 2006; Marsh, Gerlach, Trautwein, Lüdtke y Brettschneider, 2007; Valentine, Dubois y Cooper, 2004).

Como el $C A F$ fue creado y baremado por los autores con adolescentes procedentes del norte de España, se llevó a cabo un análisis de sus propiedades psicométricas con una muestra de estudiantes del sureste del país. Los resultados ponen de manifiesto que el $C A F$ muestra adecuados índices de fiabilidad y de discriminación, así como unos índices de ajuste aceptables del modelo con seis factores que coinciden con los planteados por Goñi et al. (2006). Por otra parte, el cuestionario logra un mejor ajuste y una mayor capacidad de discriminación si se elimina el elemento 32 (no tengo demasiadas cualidades como persona). En el resto de las dimensiones hay coincidencia con los resultados obtenidos por Goñi et al. (2006). La solución completamente estandarizada obtenida avala la validez de constructo del cuestionario, en tanto que las ligeras discrepancias observadas pueden achacarse a diferencias sociales o culturales de los participantes. En todo caso, la validación de un cuestionario es un proceso lento y continuo, por lo que, los resultados deben ser contrastados en otras muestras procedentes de otros lugares.

De los resultados del análisis de correlación se deduce, en primer lugar, que todas las di- mensiones evaluadas por el CAF presentan correlaciones positivas y estadísticamente significativas con el autoconcepto físico; en segundo lugar, que de todas las dimensiones evaluadas, la condición física, y el atractivo físico no parecen relacionarse con el autoconcepto general; en tercer lugar, solo la condición física, el atractivo físico y la fuerza correlacionan con la calificación en el área de Educación Física (aunque la magnitud de tales relaciones es baja), y, en cuarto lugar, todas las dimensiones o se relacionan negativamente, o las relaciones no son significativas, en el caso de la calificación global.

Los resultados del análisis de vías confirmaron casi todas las inferencias teóricas planteadas. Primero, la relación directa entre todas las dimensiones (habilidad física, condición física, fuerza y atractivo físico) con el autoconcepto físico. Segundo, la relación directa de la habilidad física y del autoconcepto físico con el autoconcepto general. Tercero, la relación directa entre la condición física y la calificación en Educación Física, y, por último, la relación directa y positiva de la calificación en Educación Física con la calificación global, y negativa de la fuerza, la condición física y el autoconcepto general con la calificación global.

De todos los resultados llama la atención la relación negativa que se observa entre fuerza, condición física, autoconcepto general y la calificación global que parece indicar que los alumnos con altas puntuaciones en esas dimensiones son objeto de calificaciones globales más bajas. Parece ser que los alumnos que se perciben con más fuerza, con mejor condición fisica y con mayor autoconcepto general provocan en el profesorado una respuesta a la baja en sus notas, tal vez al despertar expectativas de rendimiento que luego no son corroboradas con los resultados, y ésta es una vía a seguir investigando en el futuro. También llama poderosamente la atención que todas las dimensiones del autoconcepto físico interactúen positivamente con la calificación en Educación Física, de ello podemos extraer la aplicación práctica de intentar promover en las clases de Educación Física un trabajo más específico sobre dichas dimensiones mediante la implicación en actividades físicodeportivas (Boyd y Hrycaiko, 1997; Moreno et al., 2008 ) lo cual redundará en beneficios para la salud de los adolescentes (Lindwall y Hass- 
men, 2004) y en aumentos del rendimiento académico en el área.

Se necesitan más estudios, tanto sobre las cualidades del CAF como sobre las relaciones de las dimensiones que éste evalúa con el rendimiento académico, ya sea el específico del área de Educación Física como el global de todas las áreas.

\section{Referencias}

Arkin, H., y Colton, R. (1962). Tables for statiscians. Nueva York: Barnes and Noble.

Asci, F. H. (2005). The construct validity of two pshysical self-concept measures : An example fron Turkey. Psychology of Sport and Exercise, 2, 1-11.

Asci, F. H., Asci, A., y Zorba, E. (1999). Cross-cultural validity and reliability of Physical Self-Perception Profile. International Journal of Sport Psychology, 30, 399-406.

Atienza, F. I., Balaguer, I., Moreno, Y., y Fox, K. R. (2004). El perfil de autopercepción física: propiedades psicométricas de la versión española y análisis de la estructura jerárquica de las autopercepciones físicas. Psicothema, 16(3), 461-467.

Boyd, K. R., y Hrycaiko, D. W. (1997). The effect of a physical activity intervention package in the selfesteem of preadolescent and adolescent females. Adolescence, 32, 693-709.

Byrne, B. M. (1996). Measuring self-concept across the life span: Issues and Instrumentation. Washington: APA.

Coopersmith, S. (1967). The antecedents of Self-esteem. San Francisco: Freeman.

Cox, R. H. (2009). Psicología del Deporte: conceptos y sus aplicaciones ( $6^{a} E d$.). Madrid: Panamericana.

Dieppa, M., Machargo, J., Lújan, I., y Guillén, F. (2009). Autoconcepto general y físico en jóvenes españoles y brasileños que practican actividad física versus no practicantes. Revista de Psicología del Deporte, 17(2), 221-239.

Dunton, G. F., Jamner, M. S., y Cooper, D. M. (2003). Physical self-concept in adolescent girls: behavioral and physical correlation. Research Quarterly for Exercise and Sport, 74, 360-365.
Elexpuru, I., y Villa, A. (1992). Autoconcepto y Educación. Teoría, medida y práctica pedagógica. Vitoria-Gasteiz: Gobierno Vasco.

Esnaola, I. (2008). El autoconcepto físico durante el ciclo vital. Anales de Psicología, 24(1), 1-8.

Esnaola, I., Goñi, A., y Madariaga, J. M. (2008). El autoconcepto: perspectivas de investigación. Revista de Psicodidáctica, 13(1), 179-194.

Fox, K. R. (1997). The physical self. From motivation to well-being. Champaign, IL: Human Kinetics.

Fox, K. R. (2000). The effects of exercise on self-perceptions and self-esteem. En S. J. H. Biddle, K. R. Fox y S. H. Boutcher (Eds.), Physical activity and psychological well-being (pp. 88-108). London: Routhledge and Kegan Paul.

Fox, K. R., y Corbin, C. B. (1989). The Physical SelfPerception Profile: development and preliminary validation. Journal of Sports and Exercise Psychology, 11, 408-430.

García, M. S., González-Pienda, J. A., Núñez, J. C., González-Pumariega, S., Álvarez, L., Roces, C., González, R., y Valle, A. (1998). El cuestionario de metas académicas (C.M.A.). Un instrumento para la evaluación de la orientación vocacional de los alumnos de Educación Secundaria. Aula Abierta, 71, 171-199.

Goñi, A. (2009)(Coord). El autoconcepto físico. Psicología y Educación. Madrid:Pirámide.

Goñi, A., Palacios, S., Zulaika, L. M., Madariaga, J. M., y Ruiz de Azúa, S. (2002). El autoconcepto físico de los escolares entre 10 y 14 años. Versión del CYPSPP. (Informe de investigación inédito).

Goñi, A., y Rodríguez, A. (2007). Variables associates with the risk of eating disorders in adolescence. Salud Mental, 30(4), 16-23.

Goñi, A., Ruiz de Azúa, S., y Liberal, I. (2004). El autoconcepto físico y su medida. Propiedades psicosométricas de un nuevo cuestionario para la medida del autoconcepto físico. Revista de Psicología del Deporte, 13(2), 195-213.

Goñi, A., Ruiz de Azúa, S., y Rodríguez, A. (2004). Deporte y autoconcepto físico en la preadolescencia. Revista Apunts. Educación Física y Deportes, 77, 18-24.

Goñi, A., Ruiz de Azúa, S., y Rodríguez, A. (2006). Cuestionario del Autoconcepto Físico. Manual. Madrid: EOS. 
Goñi, A., y Ruiz de Azúa, S. (2009). La estructura interna del autoconcepto físico. En A. Goñi (Coord.), El autoconcepto físico: psicología y educación (pp. 81-97). Madrid: Pirámide.

Goñi, A., y Zulaika, L. M. (2000). La participación en el deporte escolar y el autoconcepto en escolares de 10/11 años de la provincia de Guipúzcoa. Revista Apunts. Educación Física y Deportes, 59, 6-10.

Gutiérrez, M., Moreno, J. A., y Sicilia, A. (1999). Medida del autoconcepto físico: una adaptación del PSPP de Fox (1990). IV Congrés de les Ciències de l'esport, l'Educaciò Física y la Recreació. Lleida: INEFC.

Harter, S. (1988). Manual for the Self-Perception Profile for Adolescents. Denver: University of Denver.

Infante, G., y Zulaika, L. M. (2009). Actividad física y autoconcepto físico. En A. Goñi (Coord.), El autoconcepto físico: psicología y educación (pp. 125153). Madrid: Pirámide.

Jöreskog, K. G. (2001). Analysis of ordinal variables 2: Cross-Sectional data. Text of the workshop, Structural Equation Modelling with LISREL 8.51. Jena: Friedrich-Schiller-Universität Jena.

León, O. G., y Montero, I. (1998). Diseños de investigación. Madrid: McGraw-Hill.

Lindwall, M., y Hassmen, P. (2004). The role of exercise and gender for physical self-perceptions and importance tatings in Swedish university students. Scandinavian Journal of Medicine \& Science in Sport, 14, 373-380.

Maïano, C., Ninot, G., y Bilard, J. (2004). Age and gender effects on global self-esteem and physical self-perception in adolescents. European Physical Education Review, 10, 53-69.

Marsh, H. W. (1990). The structure of academic selfconcept: the Marsh/Shavelson model. Journal of Educational Psychology, 82, 623-636.

Marsh, H. W. (1997). The meaasurement of physical self-concept: a construct validation approach. En K. R. Fox (Ed.), The physical self (pp. 27-58). Leeds: Human Kinetics.

Marsh, H. W., Asci, F. S., y Tomás, L. M. (2002). Multitrait-multimethod analyses of two physical selfconcept instruments: A cross-cultural perspective. Journal of Sport and Exercise Psychology, 24, 99119.
Marsh, H. W., Chanal, J. P., y Sarrazin, P. G. (2006). Self-belief does make a difference: A reciprocal effects model of the causal ordering of physical self-concept and gymnastics performance. Journal of Sports Sciences, 24(1), 101-111.

Marsh, H.W., Gerlach, E., Trautwein, U., Lüdtke, O., y Brettschneider, W. D. (2007). Longitudinal study of preadolescent sport self-concept and performance: Reciprocal effects and causal ordering. Child Development. 78(6), 1640-1656.

Marsh, H. W., y Hattie, J. (1996). Theoretical perspectives on the structure of self-concept. In B. A. Bracken (Ed.), Handbook of Self-Concept: Developmental, social, and clinical considerations (pp. 38-90). Nueva York: Wiley.

Marsh, H. W., Richards, G. E., Johnson, S., Roche, L., y Tremayne, P. (1994). Physical Self-Description Questionnaire: Psychometric properties and a multitrait-multimethod analysis of relation to existing instruments. Journal of Sport and Exercise Psychology, 16, 270-305.

Moreno, Y. (1997). Propiedades psicométricas del Perfil de Autopercepción Física (PSPP). Tesis Doctoral no publicada. Valencia: Universidad de Valencia.

Moreno, J. A., y Cervelló, E. (2005). Physical self-perception in Spanish adolescents: effects of gender and involvement in pshysical activity. Journal of Human Movement Studies, 48, 291-311.

Moreno, J. A., Cervelló, E., y Moreno, R. (2008). Importancia de la práctica físico-deportiva y del género en el autoconcepto físico de los 9 a los 23 años. International Journal of Clinical and Health Psychology, 8(1), 171-183.

Núñez, J. C., y González-Pienda, J. A. (1994). Determinantes del rendimiento académico. Variables cognitivo-emocionales, atribucionales, uso de estrategias y autoconcepto. Oviedo: Servicio de publicaciones de la Universidad de Oviedo.

Petrakis, E., y Bahls, V. (1991). Relation of physical education to self-concept. Perceptual and Motor Skills, 73, 1027-1031.

Shavelson, R. J., Hubner, J. J., y Stanton, J. C. (1976). Self concept: validation of construct interpretations. Review of Educational Research, 46, 407441.

Skaalvik, E. M., y Hagtvet, K. A. (1990). Academic achievement and self-concept: an analysis of cau- 
sal predominante in a developmental perspectiva. Journal of Personality and Social Psychology, 58(2), 292-307.

Tomás, I. (1998). Equivalencia psicométrica de una traducción del cuestionario de autoconcepto físico PSDQ (Physical Self-Description Questionnaire) al castellano. Tesis doctoral no publicada. Valencia: Universidad de Valencia.
Valentine, J. C., Dubois, D. L., y Cooper, H. (2004). The relation between self-beliefs and academic achievement: A meta-analytic review. Educational Psychologist, 39(2), 111-133.

Whitehead, J. R. (1995). A study of children's selfperceptions using a adapted Physical Self-Perception questionnaire. Pediatric Exercise Science, 7, 133-152. 